\title{
ENSURING PATIENT-CENTERED ACCESS TO CARDIOVASCULAR DISEASE MEDICINES IN LOW- AND MIDDLE-INCOME COUNTRIES THROUGH HEALTH- SYSTEM STRENGTHENING
}

Authors:

Full name:

Dan N. Tran, PharmD

Degree:

Doctor of Pharmacy

Affiliation:

Purdue University College of Pharmacy, Indiana, USA

Email:

tran.nk.tina@gmail.com

Mailing address: $\quad$ PO Box 5760 Eldoret 30100, Kenya

Disclosure statement: The author does not have any commercial, financial conflicts of interests, or any funding sources to declare.

Full name:

Degree:

Affiliation:

Email:

Mailing address:

Disclosure statement:

\section{Benson Njuguna, BPharm}

Bachelor of Pharmacy

Moi Teaching and Referral Hospital, Eldoret, Kenya

njugunaben1@gmail.com

PO Box 4606 Eldoret 30100, Kenya

The author does not have any commercial, financial conflicts of interests, or any funding sources to declare.
Full name:

Degree:

Affiliation:

Email:

Mailing address:

Disclosure statement:
Timothy Mercer, MD, MPH

Doctor of Medicine, Master of Public Health Indiana University School of Medicine, Indiana, USA

tim.merc@gmail.com

PO Box 5760 Eldoret 30100, Kenya

The author does not have any commercial, financial conflicts of interests, or any funding sources to declare.
Full name:

Degree:

Affiliation:

Email:

Mailing address:

Disclosure statement:
Imran Manji, BPharm

Bachelor of Pharmacy

Moi Teaching and Referral Hospital, Eldoret, Kenya

imranmanji@hotmail.com

PO Box 4606 Eldoret 30100, Kenya

The author does not have any commercial, financial conflicts of interests, or any funding sources to declare.
Full name:

Degree:

Affiliation:

Email:

Mailing address:

Disclosure statement:

Full name:

Degree:
Lydia Fischer, BA

Bachelor of Fine Arts in Visual Communication Design Indiana University School of Medicine

lydfisch@umail.iu.edu

941.5 Prospect Street, Indianapolis, IN 46203, USA

The author does not have any commercial, financial conflicts of interests, or any funding sources to declare.

Marya Lieberman, PhD

Doctor of Philosophy 
Affiliation:

University of Notre Dame, Department of Chemistry and

Biochemistry

Email:

mlieberm@nd.edu

Mailing address: 250 Nieuwland, Department of Chemistry and Biochemistry, University of Notre Dame, Notre Dame IN 46556

Disclosure statement: The author has received funding for this project from the Bill \& Melinda Gates Foundation, US-AID DIV program, and Indiana CTSI program.

Full name: $\quad$ Sonak Pastakia, PharmD, BCPS, MPH

Degree:

Doctor of Pharmacy, Board Certified Pharmacotherapy

Specialist, Master of Public Health

Affiliation: $\quad$ Purdue University College of Pharmacy

Email: $\quad$ spastaki@gmail.com

Mailing address: $\quad$ PO Box 5760 Eldoret 30100, Kenya 
Cardiovascular disease (CVD) is the leading cause of global mortality and is expected to reach 23 million deaths by $2030.80 \%$ of CVD deaths occur in low- and middle- income countries (LMICs). Although CVD prevention and treatment guidelines are available, translating these into practice is hampered in LMICs by inadequate healthcare systems which limit access to lifesaving medications. In this review article, we describe the deficiencies in the current LMIC supply chains that limit access to effective CVD medicines, and discuss existing solutions that are translatable to similar settings in order to address these deficiencies.

\section{Key Words}

patient-centered access, cardiovascular disease medicines, availability, accountability, adherence, health-system strengthening, low- and middle-income countries, falsified and substandard medicines

\section{Key Points}

1. $80 \%$ of deaths due to cardiovascular disease (CVD) occur in low- and middleincome countries (LMICs).

2. Translating available CVD prevention and treatment guidelines into practice is hampered in LMICs by inadequate supply chain systems which limit access to lifesaving medicines.

3. We propose three barriers (' $3 \mathrm{~A}$ ' challenges) to patient-centered access to essential CVD medicines: dismal availability to medicines, lack of accountability in the supply chain, and poor medication adherence.

4. Many challenges with CVD medication access relate to on-the-ground challenges. Pilot programs demonstrated improvement in CVD medication 
access by standardizing supply chain management, improving human resource efficiency, and extending supply chain considerations to improve patient adherence.

5. Evaluating the health impact and cost effectiveness of these existing solutions will be crucial for scaling up and ultimately providing patient-centered access to CVD medicines in LMICs. 


\section{INTRODUCTION}

Cardiovascular disease (CVD) is the leading cause of global mortality and is expected to reach 23 million deaths by $2030 .^{1,2} 80 \%$ of CVD deaths occur in low and middle income countries (LMICs) and predominantly affects a younger population compared to high income countries (HICs), thus having a significant impact on the economic growth of LMICs. ${ }^{1,3}$ The mortality burden from CVD is projected to increase in LMICs alongside the lifestyle associated epidemiologic shift favouring non-communicable disease development ${ }^{4}$. The associated economic losses are also expected to reach billions of dollars over the next decade, representing yet another barrier to development for LMICs. ${ }^{3}$ Although CVD prevention and treatment guidelines are available, translating these into practice is hampered in LMICs by inadequate healthcare systems with limited access to potentially lifesaving medications. ${ }^{5}$

Enumerating the barriers to access to CVD medicines requires an examination of both the 'policy level' barriers as well as 'on-the-ground' issues. At the policy level, limited national funding due to competing health priorities, slow incorporation of CVD drugs into the essential medicines list (EML), and structural and financial barriers all limit access to CVD drugs and have been discussed previously with recommendations proposed. ${ }^{6,7}$ Advocacy to create awareness among policy makers on the threat of CVDs to LMIC populations' health and economic prosperity will increase funding and foster equitable access through inclusion of more CVD drugs into the EML. Overcoming legal barriers in patent law will expedite generic availability to increase affordability with streamlined global and local procurement practices further bringing down cost of CVD drugs in the public 
sector. Finally, engaging the commercial sector in order to regulate mark-up on CVD drugs will minimize out-of pocket expense for patients. ${ }^{6,7}$

Despite the frequent emphasis on policy level considerations for increasing CVD medication access, the rate limiting steps rest within the healthcare system's supply chain. This is the focus of our review paper. ${ }^{3,8}$ Dismal drug availability for medicines for the prevention and treatment of CVD in public facilities in LMICs forces patients to turn to private chemists where costs of medication are often unaffordable. $^{9-11}$ Systemic deficiencies and inefficiencies in medicines' regulation and distribution have led to a rampant counterfeit medicines burden in LMICs. ${ }^{12}$ These deficiencies together contribute to the mortality and morbidity imposed on LMIC populations. $^{13}$

In this review article, we describe the deficiencies in the current LMIC supply chains that limit access to effective CVD medicines, and discuss existing solutions that are translatable to other low-resource settings to address these deficiencies.

\section{CHALLENGES}

We identified three primary barriers (' $3 A$ ' challenges) in the healthcare system supply chain which co-exist to limit access to essential CVD medicines (Figure 1.) The challenges are dismal availability to medicines, lack of accountability in the supply chain, and poor medication adherence.

\section{Availability}

A reliable supply chain system is crucial to achieve consistent availability of essential medicines. ${ }^{14}$ Patients with CVD, or at risk for CVD, often require lifelong medicines for the treatment and prevention of CVD events. Therefore, functional CVD medicine supply chains are crucial to improve global cardiovascular health. 
Unfortunately, several analyses demonstrated that availability of CVD medicines across many LMIC settings is sub-optimal. ${ }^{7}$ In 2007 , a cross-sectional study investigating CVD medicines in 6 LMICs revealed a less than $7.5 \%$ availability of medicines in the public sector. ${ }^{10}$ In 2011 , World Health Organization/Health Action International data of 36 countries revealed that the overall availability of these medicines was still poor, representing a mean availability of $26 \%$ in the public sector. ${ }^{15}$ Recently, the 2016 Prospective Urban Rural Epidemiology (PURE) study showed availability of CVD meds ranged from $3 \%-73 \%$ and $25 \%-80 \%$ in rural and urban LMIC settings respectively. ${ }^{16}$

To understand the persistently low availability rates of CVD medicines in LMICs, we analyze practical challenges with a focus on the public sector's supply chains that lead to weak supply chain design, poor operating performance, and low access for patients who these medicines.

Within the public sector of most LMICs, the government procures medications and distributes them to other health facilities using the government Central Medical Store (CMS). ${ }^{17,18}$ The CMS is responsible for distributing medicines to district facilities, and they, in turn, supply medicines to the sub-district clinics. ${ }^{17,18}$ One of the challenges faced in these settings is the unnecessary complexity of supply chain design, since most LMICs include multiple tiers of stock management before medicines can reach the intended patient. ${ }^{17,18}$ Because the CMS is only responsible for distributing medicines to district facilities, other sub-district facilities may fall under the supervision of other departments in the Ministry of Health. Diffuse accountability - defined as "fragmentation of responsibility and governance" - occurs when this supply chain structure is followed, as exemplified in countries like Ethiopia, Kenya, Mozambique and Zambia. ${ }^{17}$ Together with poor communication, these factors lead to 
long intervals between medicine resupply and distorted information about patient demand. $^{19}$

Poor operational management also accounts for the low availability of medicines in the public sector. There is insufficient interest in investing in operating costs such as drug distribution, leading to medicines not being delivered to designated health facilities in a timely manner. ${ }^{17,20}$ The inability to capture up-to-date consumption data also prohibits adequate planning for procurement and resupply. ${ }^{20}$ Additionally, the lack of evaluation and feedback for supply chain management staff creates minimal incentives for performance improvement and requires little accountability in job performance. ${ }^{17,20}$

\section{Accountability}

Lack of accountability in the LMIC supply chain has hampered access to high quality medicines for CVD. ${ }^{17,21,22}$ In this section, we focus on (1) the corrupt system dynamics which propagate falsified and substandard medicines (FSM), and (2) the lack of oversight over pharmacy-related human resources for health.

\section{Corrupt System Dynamics Propagating FSMs}

The many checks and balances typically found in the different levels of the supply chain in HICs include adequate regulatory oversight of manufacturers and distributors, adequate law enforcement, and adequate surveillance systems. These however, are largely absent in LMICs. ${ }^{12}$ This lack of accountability allows FSMs to penetrate the supply chain. Substandard medicines contain incorrect levels of active pharmaceutical ingredients (API), while falsified ones contain unapproved ingredients and may lack the API entirely. ${ }^{12}$ FSMs cause significant harm to vulnerable populations that are forced to rely on a compromised supply chain. Although a majority of reports of the harm done by FSMs have focused on anti- 
infective FSMs, ${ }^{23-25}$ it is now apparent that CVD medicines have become a prime FSM target. ${ }^{26}$ In a cross-sectional analysis evaluating the quality of anticoagulation (acenocoumarol), hypertension (amlodipine, atenolol, captopril, furosemide and hydrochlorthiazide), and dyslipidemia (simvastatin) medications in 10 countries in Africa, $16.3 \%$ of the sampled CVD medications were found to be of poor quality, ranging from $0-30 \%$ depending on the drug and type (brand versus generic). Generic medicines had 10 times lower quality than brand medicines, with $30 \%$ of generic captopril and amlodipine being of poor quality. No generic acenocoumarol or hydrochlorthiazide was found to be of poor quality in this study. ${ }^{26}$

Gaps in the health system that lead to FSMs are summarized in Figure 2. In the past two decades, there has been an increasing number of manufacturers and distributors supplying medicines to LMICs without a matching increase in the regulatory infrastructure in the industry. From 1995 to 2006, there was a $50 \%$ growth in spending for pharmaceuticals worldwide. ${ }^{18}$ This increase in demand has been met with a dramatic increase in the production of medications, especially in LMICs like India where the pharmaceutical industry is valued at over $\$ 24$ billion. ${ }^{18}$ Despite this, public health expenditure in sub-Saharan Africa was still below the $15 \%$ expenditure recommended by the WHO's Abuja Declaration, resulting in a constrained pool of resources allocated to regulatory activities ${ }^{27}$ Furthermore, as wholesalers and distributors purchase medicines from a wide variety of sources at the lowest cost, including informal suppliers who typically circumvent the registration process, ${ }^{28}$ they risk buying medicines of compromised quality. ${ }^{26}$ Additionally, reports have highlighted the high potential for corruption as different stakeholders try to circumvent the numerous steps involved with the procurement process between manufacturers, distributors, and chemists. ${ }^{29}$ Because LMIC chemists preferentially 
stock the cheapest generic options available from distributors and wholesalers, as opposed to higher cost branded CVD generics, LMIC settings frequently only access the lowest priced generics available in the market. ${ }^{15,16}$ This dynamic exposes patients in the lower socioeconomic classes to the harmful consequences of FSMs with little potential for remediation via typically unaffordable and inefficient legal channels. $^{30}$

\section{Human Resources for Health $(H R H)$}

The lack of healthcare workers has been described as one of the main drivers of limited access to care and suboptimal care delivery for chronic diseases in LMICs. The pharmacy workforce is an example of this; the lower the World Bank Income Status Classification of a country, the fewer pharmacists and pharmacies are present to serve the population. ${ }^{31}$ This lower density of care providers and pharmacy access points in LMICs is unable to reliably provide access to CVD medicines to the populations they serve. This lack of access to care is further plagued by "absenteeism" among pharmacists. ${ }^{32}$ In a detailed evaluation conducted in Machakos, Kenya, it was found that pharmacists were the most absent healthcare cadre with an absenteeism rate of $42 \% .{ }^{33}$ Finally, limited supervision of the HRH workforce represents another aspect of limited accountability, particularly in the public healthcare sector. With many public sector staff having their own private healthcare clinics, pilferage of public sector commodities and diversion to private businesses can significantly reduce their operating costs and improve their profits. ${ }^{21,22}$ The lack of accountability described in this section highlights the current supply chain dynamics where the different stakeholders consistently prioritize their own interests above the interests of the patient. As we continue to push for more impactful service delivery, we must shift to a more patientcentered approach. 


\section{Adherence}

Functional medicine supply chains are fundamental to ensuring access to health care services in LMICs. ${ }^{14,34}$ Traditionally, access has been narrowly defined as the ability or opportunity of a population to enter into the health care system and be able to obtain health care goods and services. ${ }^{35,36}$ This defines access solely from the health system perspective, with a focus on "supply" side factors. In this view, a medicine supply chain may be deemed to be successful in providing access to essential medicines for a population, by making medicines available and affordable, while ignoring a host of "demand" side, patient-level factors including medication utilization and adherence. ${ }^{35,37-39}$ This approach leaves the most important stakeholder - the patient - out of the picture when it comes to designing, implementing and evaluating supply chains. If we are to reach our goals of reducing the burden of CVD in LMICs, then ensuring the uptake and adherence by patients must be part of our evaluation framework. Within this framework, the successful supply chain does not end with the delivery of essential medicines to the pharmacy or clinic; it ends with the patient actually taking those medicines consistently and realizing improved health outcomes.

Combination therapy with essential CVD medicines reduces primary and secondary coronary heart disease events by $80 \% .{ }^{40}$ Unfortunately, adherence to these medicines is low. When assessing the appropriateness of prescriptions for CVD medications from the healthcare provider perspective, studies have shown that there may be a low level of uptake of evidence-based recommendations by clinicians. In the WHO Prevention of Recurrences of Myocardial Infarction and Stroke (PREMISE) study, evidence-based medicines for secondary prevention of CVDs were inadequately prescribed in LMICs. ${ }^{41}$ Aspirin was only prescribed for 
$79.6 \%$ of patients, beta-blockers for $44.2 \%$, angiotensin-converting enzyme inhibitors for $39.5 \%$, and statins for $19.6 \%$. Another recent study found that heart failure treatment patterns in rural Kenya are poor, with only $55 \%$ of patients with dilated cardiomyopathy taking angiotensin-converting enzyme inhibitors, $45 \%$ taking betablockers, and $23 \%$ taking digoxin. ${ }^{42}$ A systematic review that examined barriers to hypertension treatment by healthcare providers in LMICs reported that the most frequently-encountered health system barriers were lack of equipment, space, medicine, and staff. ${ }^{43}$

There are five main reasons that patients do not adhere to medication regimens: socioeconomic, medication-related, condition-related, health systemrelated, and patient-related factors. In LMICs, socioeconomic factors (i.e. cost of medications) and medicine-related factors (i.e. pill burden and side effects) are particularly salient in determining adherence to CVD medicines. ${ }^{44}$ Three potential costs - transportation, user fees to see the clinician, and the costs of the medicines themselves - all hinder patients from adhering to essential CVD medicines. The first and most obvious cost barrier, is the price the patient pays to buy the medicine at the pharmacy. In LMICs, high prices of medicines have clearly been shown to decrease patient purchase and therefore, uptake and adherence to essential medicines. ${ }^{10,15,43,45}$ Transportation costs are also a major barrier to patients accessing care, particularly in rural areas. ${ }^{43,46}$ Additionally, many patients in LMICs have to pay out-of-pocket user fees just to see clinicians, even in the public sector, which further increases the cost of accessing care. ${ }^{47,48}$

The complexity of the treatment regimen for primary and secondary prevention of CVDs can be overwhelming to many patients. The daily number of prescribed pills as well as the frequency at which patients must remember to take 
their medicines are recognized as factors responsible for patients' lack of adherence. ${ }^{44,49}$ Additionally, besides availability, medications' side effects have been found to be the most common barrier to patient's adherence to treatment. When translated to clinical significance, side effects had a two-fold increased risk of nonadherence. $^{43}$

\section{EXISTING SOLUTIONS}

In order to address existing deficiencies in the LMIC supply chain, programs to ensure access to CVD medicines have been established. This section will highlight examples that have evaluated access to CVD and non-CVD medicines from both the supply and demand perspectives, with measurable patient-centered outcomes.

\section{Availability}

The concept of "revolving drug funds" (RDF) dates back to 1989 when an RDF project was initiated in Ghana. ${ }^{50}$ In an RDF, seed funding is used to purchase an initial stock of medicines, which are then sold at a price point sufficient to support staff salaries and replace the initial stock. Building on the experiences and successes of RDF projects in other LMICs, ${ }^{51,52}$ the Academic Model Providing Access To Healthcare (AMPATH) program in western Kenya created a network of revolving fund pharmacies (RFPs) in 2011 to address availability of affordable essential medicines. RFPs serve as "back-up pharmacies" when government pharmacies are unable to supply essential medicines, including CVD drugs, to patients. ${ }^{53}$ Medicines are sold by RFPs with a small mark-up (10\%), and the net profit earned is used to ensure an uninterrupted source of drugs. To ensure that medicines are consistently available and the patients' demand is met, RFPs conduct 
regular needs-assessments (i.e. frequent stock-take audits) to forecast usage and ensure accountability in their daily operations. They also developed a waiver system to enable access to indigent patients, and expanded to multiple geographical locations to meet patients' needs. Findings from this pilot study showed an increase of essential medicine availability to greater than $90 \%$, with $40 \%$ of sales being attributed to chronic disease medicines.

Other efforts to address availability of non-CVD medicines, which can be adopted to improve CVD medicines availability, have also been described in the literature. New and simple communication methods have been tested to monitor and distribute stocks of medicines, specifically in rural settings. ${ }^{54}$ The "SMS for Life" program in rural Tanzania is an example of a replicable technological advancement used to improve supply of anti-malarial medicines. ${ }^{54}$ Using widely available SMS technology, the implementers created an inventory tracking application that could geographically map medicine availability at all facilities in their network. With this system, medicine stock-outs were reduced from $78 \%$ to $26 \% .{ }^{54}$ Implementation and evaluation of health information systems to improve real-time communication of consumption rate, inventory, and procurement status should be encouraged in order to improve availability of medicines.

\section{Accountability}

To address the quality assurance of medicines in LMICs, several technologies have been developed to detect FSMs in these settings, including methods to analyze drug formulations. ${ }^{55}$ Technologies suitable for LMICs allow for detection of correct API at a low cost, require no sample preparation, and can be performed outside a laboratory setting. A practical example of how these technologies may be carried out is an ongoing multi-country evaluation of Paper Analytical Devices, also known 
as PADs. PADs are paper cards that carry out a library of colorimetric tests to rapidly and qualitatively detect falsified medicines (Figure 3). Studies using PADs to test for low-quality antimalarial, antimicrobial, and anti-tuberculosis medicines have confirmed the high sensitivity and specificity of these devices. ${ }^{56}$ To address the growing problems with CVD medicines, PADs have recently been formulated to test for commonly used CVD medications. Confirmatory testing with standard instrumental methods is used to follow up on suspicious products and detect FSMs.

Task shifting has been suggested as an important strategy to address the $\mathrm{HRH}$ shortage in LMICs that hampers CVD management. ${ }^{57}$ Task shifting is described as the rational redistribution of tasks within the health workforce in order to more efficiently use the limited $\mathrm{HRH}$ to improve patient care. ${ }^{58}$ To date, four randomized controlled trials conducted in LMICs have demonstrated the effectiveness of using non-physician health workers to manage patients with postacute coronary syndrome, hypertension, or diabetes. ${ }^{59}$ These program, led by clinically trained nurses, pharmacists, and community-health workers, demonstrated an improvement in adherence to life-saving CVD medicines, a significant reduction in blood pressure, and a reduction in glycated haemoglobin at a fraction of the cost anticipated with physician-based health care delivery. ${ }^{60-63}$ Another study examining linkage and retention to hypertension care using community health workers in Kenya is in progress. ${ }^{64}$

\section{Adherence}

Holistic, patient-centered interventions have been shown to improve medication adherence. For HIV, medication delivery by peer counsellors was found to be more effective in improving adherence than directly observed antiretroviral therapy. ${ }^{65}$ Similarly, in managing CVDs, the use of a multidisciplinary team of other 
non-physician care providers demonstrated improved medication adherence and reduction in low-density lipoprotein cholesterol. ${ }^{66}$

To address adherence as one of the dimensions of supply chain management, several trials examined the effects of improved adherence on strengthening access to CVD medicines. The use of "polypills", also known as a fixed-dose, combination regimen, directly addresses the adherence barriers of regimen complexity and pill burden, and have been shown to increase patient adherence and reduce CVD events in patients with CVDs or who are at risk for CVDs. ${ }^{67,68}$

A few studies have also shown discrepancies between evidence-based recommendations for heart failure and clinicians' prescribing patterns. ${ }^{43}$ Strategies that have been proven to change health care professional behaviours and patient outcomes include: financial incentives, audit and feedback, automated reminders, and educational outreach visits. ${ }^{69}$ Using innovative models to address adherence from both the prescriber and patient standpoint is arguably one of the most effective solution to improve patient-centred access to CVD medicines in LMICs.

\section{FUTURE DIRECTIONS}

CVD poses a significant public health threat to populations in LMIC that is expected to grow over the next decade. ${ }^{2}$ CVD also imposes a significant economic burden on LMIC populations. ${ }^{3}$ Improving access and uptake of CVD medicines is a crucial step to addressing this health and development barrier. As discussed in this paper, existing challenges in the LMIC CVD medication supply chain extend beyond previously described policy level issues ${ }^{6,7}$ and must include substantial 'on-theground' deficiencies. In order to address these 'on-the-ground' deficiencies and 
charter a path to improved access to CVD drugs for LMIC populations, we propose the comprehensive ' $3 A^{\prime}$ ' pathway to progress (Figure 1).

The first step is to scale up existing solutions that have already demonstrated effectiveness in addressing the ' 3 A challenges' in the LMIC supply chain. We have highlighted examples of such context-specific solutions employed in various LMIC settings and advocate that such solutions need to be scaled up to other regions and countries facing similar problems. Such solutions may need to be contextualised to address local problems and adhere to local policy frameworks in order to maximise impact, but we anticipate that the core principles and overall design will be maintained. For example, the RFP model has been demonstrated as a cost-effective solution that addresses drug availability by backing up public health facility pharmacies with affordably priced medicines. ${ }^{53}$ Such a model may be replicated in countries facing similar problems in availability and affordability with modifications to align with local rules on pricing structures, pharmacy regulation agents and governance of public-private partnerships.

Evaluation of the health impact and cost-effectiveness of these existing solutions is needed. A limitation of the discussed solutions is a lack of rigorous scientific evaluation to document their benefit over traditional supply chain models in LMICs. In resource-limited settings with many competing priorities, robust evidence that demonstrates clear patient-centered and economic benefits of an intervention would therefore be required to justify funding for scale up. As such, randomised and quasi-randomised trials should be conducted to identify solutions with the most significant health impact and cost-effectiveness. Although such efforts may require large resources up-front, we expect they would translate to cost savings through long-term health and economic improvements. 


\section{CONCLUSION}

In this review article, we described the '3A challenges' - dismal availability to essential medicines, lack of accountability in the medication supply chain, and poor medication adherence - in the current LMIC supply chains that limit access to effective CVD care. The described existing solutions to ensure access to CVD medicines in LMIC settings can and should be applied in other resource-limited settings. However, efforts to improve global access to CVD medicines must take into account health system challenges. Innovation to find new patient-centred, crosscutting, and context-specific solutions to the $3 \mathrm{~A}$ challenges is therefore required in LMICs. Such innovations should address a majority or all of the ' $3 \mathrm{~A}$ challenges' discussed in this paper, with a special emphasis on promoting patient adherence as part of the supply chain to maximize CVD outcomes. 


\section{REFERENCES}

1. Global, regional, and national age-sex specific all-cause and cause-specific mortality for 240 causes of death, 1990-2013: a systematic analysis for the Global Burden of Disease Study 2013. Lancet (London, England). 2015;385(9963):117-171.

2. Mathers CD, Loncar D. Projections of global mortality and burden of disease from 2002 to 2030. PLoS medicine. 2006;3(11):e442.

3. World Health Organization. Global status report on noncommunicable diseases 2014. Geneva: WHO; 2014.

4. Yusuf S, Reddy S, Ounpuu S, Anand S. Global burden of cardiovascular diseases: part I: general considerations, the epidemiologic transition, risk factors, and impact of urbanization. Circulation. 2001;104(22):2746-2753.

5. Fuster V, Kelly BB, Vedanthan R. Promoting Global Cardiovascular Health: Moving Forward. Circulation. 2011;123(15):1671-1678.

6. Kishore SP, Kolappa K, Jarvis JD, et al. Overcoming Obstacles To Enable Access To Medicines For Noncommunicable Diseases In Poor Countries. Health affairs (Project Hope). 2015;34(9):1569-1577.

7. Kishore SP, Vedanthan R, Fuster V. Promoting global cardiovascular health ensuring access to essential cardiovascular medicines in low- and middle-income countries. $J$ Am Coll Cardiol. 2011;57(20):1980-1987.

8. Systems for Improved Access to Pharmaceuticals and Services (SIAPS). Enhancing Health Outcomes for Chronic Diseases in Resource-Limited Settings by Improving the Use of Medicines: The Role of Pharmaceutical Care. Submitted to the U.S. Agency for International Development by the SIAPS Program. Arlington, VA: Management Sciences for Health. May 2014.

9. Cameron A, Ewen M, Ross-Degnan D, Ball D, Laing R. Medicine prices, availability, and affordability in 36 developing and middle-income countries: a secondary analysis. Lancet (London, England). 2009;373(9659):240-249.

10. Mendis S, Fukino K, Cameron A, et al. The availability and affordability of selected essential medicines for chronic diseases in six low- and middle-income countries. Bulletin of the World Health Organization. 2007;85(4):279-288.

11. Kankeu HT, Saksena $P, X u K$, Evans DB. The financial burden from noncommunicable diseases in low- and middle-income countries: a literature review. Health research policy and systems / BioMed Central. 2013;11:31.

12. Almuzaini T, Choonara I, Sammons H. Substandard and counterfeit medicines: a systematic review of the literature. BMJ Open. 2013;3(8):e002923.

13. Wirtz VJ, Kaplan WA, Kwan GF, Laing RO. Access to Medications for Cardiovascular Diseases in Low- and Middle-Income Countries. Circulation. 2016;133(21):20762085.

14. Equitable Access to Essential Medicines: A Framework for Collective Action - WHO Policy Perspectives on Medicines, No. 008, March 2004. World Health Organization, Geneva;2004.

15. van Mourik MS, Cameron A, Ewen M, Laing RO. Availability, price and affordability of cardiovascular medicines: a comparison across 36 countries using WHO/HAl data. BMC cardiovascular disorders. 2010;10:25.

16. Khatib R, McKee $\mathrm{M}$, Shannon $\mathrm{H}$, et al. Availability and affordability of cardiovascular disease medicines and their effect on use in high-income, middle-income, and lowincome countries: an analysis of the PURE study data. Lancet (London, England). 2016;387(10013):61-69.

17. Yadav P. Health Product Supply Chains in Developing Countries: Diagnosis of the Root Causes of Underperformance and an Agenda for Reform. Health Systems \& Reform. 2015;1(2):142-154.

18. The World Medicines Situation 2011, 3rd Edition. World Health Organization, Geneva, 2011. 
19. Hau L Lee, V Padmanabhan, Whang S. The Bullwhip Effect In Supply Chains. Sloan Management Review.38(Spring 1997):93-102.

20. Sarley, David, Linda Allain, Akkihal A. Estimating the global in-country supply chain costs of meeting the MDGs by 2015. Arlington, Va.: USAID | DELIVER PROJECT, Task Order 1.;2009.

21. Bateman C. Drug stock-outs: inept supply-chain management and corruption. South African medical journal = Suid-Afrikaanse tydskrif vir geneeskunde. 2013;103(9):600602.

22. Vian T. Review of corruption in the health sector: theory, methods and interventions. Health policy and planning. 2008;23(2):83-94.

23. Countering the problem of falsified and substandard drugs. Institute of Medicine of the National Academies;February 2013.

24. Nayyar GM, Breman JG, Newton PN, Herrington J. Poor-quality antimalarial drugs in southeast Asia and sub-Saharan Africa. The Lancet. Infectious diseases. 2012;12(6):488-496.

25. Newton PN, Green MD, Fernandez FM, Day NP, White NJ. Counterfeit anti-infective drugs. The Lancet. Infectious diseases. 2006;6(9):602-613.

26. M Antignac, BI Diop, D Macquart De Terline, et al. Quality assessment of 7 cardiovascular drugs in SubSaharan African countries: Results of the seven study by drug and version of drug World Congress of Cardiology and Cardiovascular Health; June 2016, Poster PS075; Mexico City, Mexico.

27. Tumusiime P, Gonani A, Walker O, Asbu EZ, Awases M, Kariyo PC. Health systems in sub-Saharan Africa: What is their status and role in meeting the health Millennium Development Goals?

28. Campos JE, Pradhan S. The Many Faces of Corruption : Tracking Vulnerabilities at the Sector Level

Washington, DC: World Bank. (c) World Bank. https://openknowledge.worldbank.org/handle/10986/6848 License: CC BY 3.0 IGO. 2007.

29. Ho PM, Rumsfeld JS, Masoudi FA, et al. Effect of medication nonadherence on hospitalization and mortality among patients with diabetes mellitus. Arch Intern Med. 2006;166(17):1836-1841.

30. Fake medicines flood Kenyan market as experts warn of looming health crisis [press release]. Daily Nation.

31. Anderson C, Roy T. FIP Global Pharmacy Workforce Report. International Pharmaceutical Federation (FIP);2012.

32. Chaudhury N, Hammer J, Kremer M, Muralidharan K, Rogers FH. Missing in action: teacher and health worker absence in developing countries. The journal of economic perspectives : a journal of the American Economic Association. 2006;20(1):91-116.

33. Absenteeism of health care providers in Machakos District, Kenya. . Institute of Policy Analysis and Research (IPAR). IPAR Policy Brief 12, no. 2. ;2008.

34. Dowling P. Healthcare Supply Chains in Developing Countries: Situational Analysis. Arlington, Va.: USAID | DELIVER PROJECT, Task Order 4.;2011.

35. Aday LA, Andersen R. A framework for the study of access to medical care. Health services research. 1974;9(3):208-220.

36. Frenk J, White KL. The concept and measurement of accessibility. PAHO. Scientific Publication: Pan American Health Organization; 1992:842-855.

37. Andersen RM. Revisiting the behavioral model and access to medical care: does it matter? Journal of health and social behavior. 1995;36(1):1-10.

38. Gulliford M, Figueroa-Munoz J, Morgan M, et al. What does 'access to health care' mean? Journal of health services research \& policy. 2002;7(3):186-188.

39. Mooney $\mathrm{GH}$. Equity in health care: confronting the confusion. Effective health care. 1983;1(4):179-185. 
40. Wald NJ, Law MR. A strategy to reduce cardiovascular disease by more than $80 \%$. BMJ (Clinical research ed.). 2003;326(7404):1419.

41. Mendis S, Abegunde D, Yusuf S, et al. WHO study on Prevention of REcurrences of Myocardial Infarction and StrokE (WHO-PREMISE). Bulletin of the World Health Organization. 2005;83(11):820-829.

42. Bloomfield GS, DeLong AK, Akwanalo CO, et al. Markers of Atherosclerosis, Clinical Characteristics, and Treatment Patterns in Heart Failure: A Case-Control Study of Middle-Aged Adult Heart Failure Patients in Rural Kenya. Global heart. 2016;11(1):97-107.

43. Khatib R, Schwalm JD, Yusuf S, et al. Patient and healthcare provider barriers to hypertension awareness, treatment and follow up: a systematic review and metaanalysis of qualitative and quantitative studies. PloS one. 2014;9(1):e84238.

44. Castellano JM, Copeland-Halperin R, Fuster V. Aiming at strategies for a complex problem of medical nonadherence. Global heart. 2013;8(3):263-271.

45. Pandey KR, Meltzer DO. Financial Burden and Impoverishment Due to Cardiovascular Medications in Low and Middle Income Countries: An Illustration from India. PloS one. 2016;11(5):e0155293.

46. Ambaw AD, Alemie GA, SM WY, Mengesha ZB. Adherence to antihypertensive treatment and associated factors among patients on follow up at University of Gondar Hospital, Northwest Ethiopia. BMC public health. 2012;12:282.

47. Bhojani $U$, Thriveni $B$, Devadasan $R$, et al. Out-of-pocket healthcare payments on chronic conditions impoverish urban poor in Bangalore, India. BMC public health. 2012;12:990.

48. Mclntyre D, Thiede M, Dahlgren G, Whitehead M. What are the economic consequences for households of illness and of paying for health care in low- and middle-income country contexts? Social science \& medicine (1982). 2006;62(4):858865.

49. Ho PM, Bryson CL, Rumsfeld JS. Medication adherence: its importance in cardiovascular outcomes. Circulation. 2009;119(23):3028-3035.

50. Garner P. The Bamako initiative. BMJ (Clinical research ed.). 1989;299(6694):277278.

51. Murakami H, Phommasack B, Oula R, Sinxomphou S. Revolving drug funds at frontline health facilities in Vientiane, Lao PDR. Health policy and planning. 2001;16(1):98-106.

52. Uzochukwu BS, Onwujekwe OE, Akpala CO. Effect of the Bamako-Initiative drug revolving fund on availability and rational use of essential drugs in primary health care facilities in south-east Nigeria. Health policy and planning. 2002;17(4):378-383.

53. Manji I, Manyara SM, Jakait B, et al. The Revolving Fund Pharmacy Model: backing up the Ministry of Health supply chain in western Kenya. The International journal of pharmacy practice. 2016.

54. Barrington J, Wereko-Brobby O, Ward P, Mwafongo W, Kungulwe S. SMS for Life: a pilot project to improve anti-malarial drug supply management in rural Tanzania using standard technology. Malaria journal. 2010;9:298.

55. Kovacs S, Hawes SE, Maley SN, Mosites E, Wong L, Stergachis A. Technologies for Detecting Falsified and Substandard Drugs in Low and Middle-Income Countries. PloS one. 2014;9(3):e90601.

56. Paper Analytical Device Project. http://padproject.nd.edu/. Accessed June 5, 2016.

57. Joshi $\mathrm{R}$, Alim M, Kengne AP, et al. Task shifting for non-communicable disease management in low and middle income countries--a systematic review. PloS one. 2014;9(8):e103754.

58. Task shifting: Rational redistribution of tasks among health workforce teams. Geneva: World Health Organization;2008.

59. Ogedegbe G, Gyamfi J, Plange-Rhule J, et al. Task shifting interventions for cardiovascular risk reduction in low-income and middle-income countries: a systematic review of randomised controlled trials. BMJ open. 2014;4(10):e005983. 
60. Adeyemo A, Tayo BO, Luke A, Ogedegbe O, Durazo-Arvizu R, Cooper RS. The Nigerian antihypertensive adherence trial: a community-based randomized trial. Journal of hypertension. 2013;31(1):201-207.

61. Mendis S, Johnston SC, Fan W, Oladapo O, Cameron A, Faramawi MF. Cardiovascular risk management and its impact on hypertension control in primary care in low-resource settings: a cluster-randomized trial. Bulletin of the World Health Organization. 2010;88(6):412-419.

62. Nesari M, Zakerimoghadam M, Rajab A, Bassampour S, Faghihzadeh S. Effect of telephone follow-up on adherence to a diabetes therapeutic regimen. Japan journal of nursing science : JJNS. 2010;7(2):121-128.

63. Xavier D, Gupta R, Kamath D, et al. Community health worker-based intervention for adherence to drugs and lifestyle change after acute coronary syndrome: a multicentre, open, randomised controlled trial. The lancet. Diabetes \& endocrinology. 2016;4(3):244-253.

64. Vedanthan $\mathrm{R}$, Kamano $\mathrm{JH}$, Naanyu $\mathrm{V}$, et al. Optimizing linkage and retention to hypertension care in rural Kenya (LARK hypertension study): study protocol for a randomized controlled trial. Trials. 2014;15:143.

65. Ford N, Nachega JB, Engel ME, Mills EJ. Directly observed antiretroviral therapy: a systematic review and meta-analysis of randomised clinical trials. Lancet (London, England). 2009;374(9707):2064-2071.

66. Shaffer J, Wexler LF. Reducing low-density lipoprotein cholesterol levels in an ambulatory care system. Results of a multidisciplinary collaborative practice lipid clinic compared with traditional physician-based care. Archives of internal medicine. 1995;155(21):2330-2335.

67. Lonn E, Bosch J, Teo KK, Pais P, Xavier D, Yusuf S. The polypill in the prevention of cardiovascular diseases: key concepts, current status, challenges, and future directions. Circulation. 2010;122(20):2078-2088.

68. Yusuf S, Lonn E, Pais P, et al. Blood-Pressure and Cholesterol Lowering in Persons without Cardiovascular Disease. The New England journal of medicine. 2016;374(21):2032-2043.

69. Beran D, McCabe A, Yudkin JS. Access to medicines versus access to treatment: the case of type 1 diabetes. Bulletin of the World Health Organization. 2008;86(8):648-649.

Figure Legends

Figure 1. The $3 A$ challenges of access to CVD medicines and the comprehensive $3 A$ pathway to progress to improve patient-centered access.

Figure 2. Gaps of LMIC supply chain that lead to FSMs.

Figure 3. Using the Paper Analytical Device (PAD) for rapid field screening of medications. 
Figure 1: The 3A challenges of access to CVD medicines and the comprehensive 3A pathway to progress to improve patient-centered access

\section{1 ' $3 A^{\prime}$ ' challenges to patient-centered access to CVD medicines}

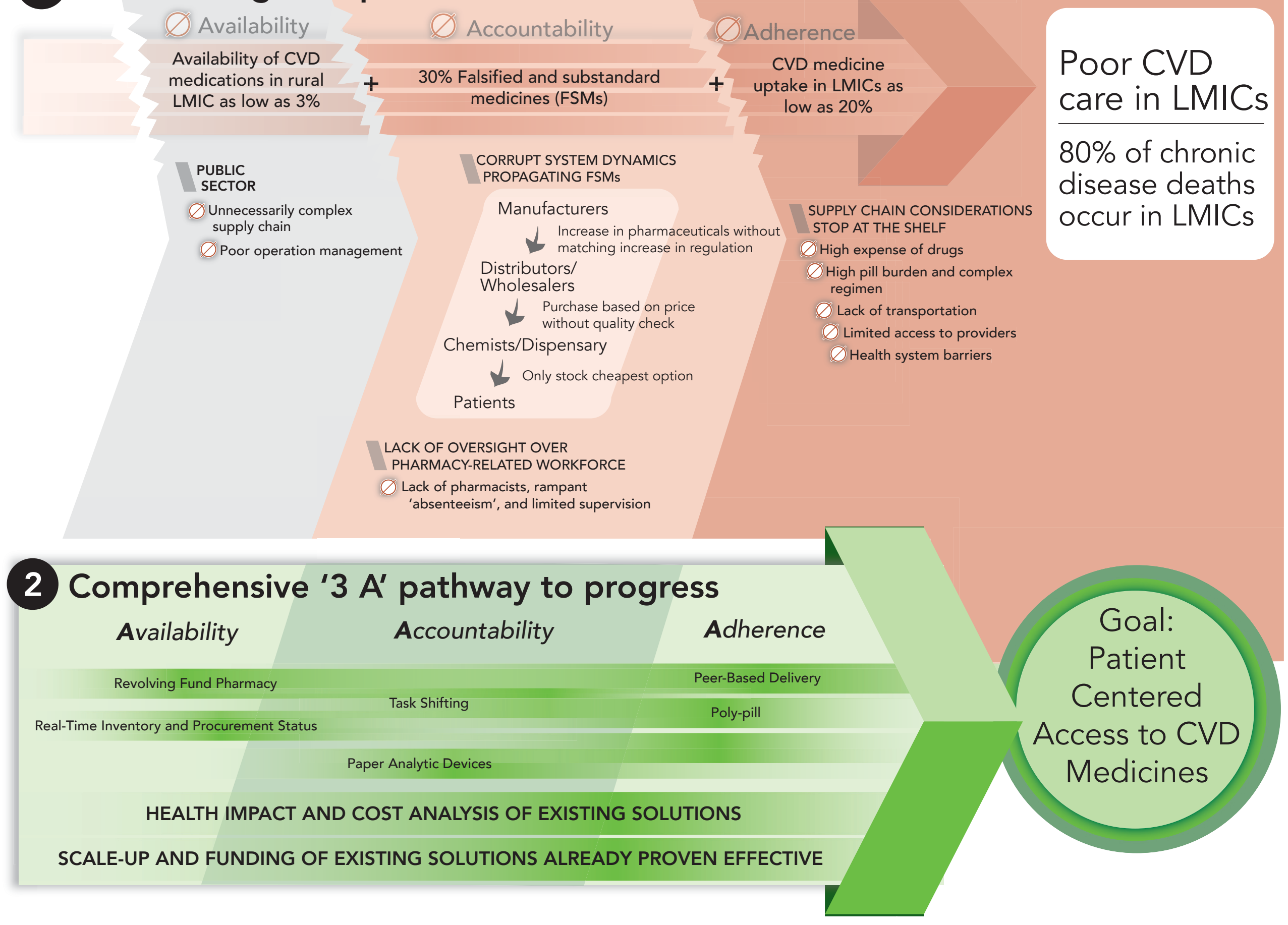


Figure 2: Gaps in LMIC supply chain that lead to FSMs

\section{Breaks in supply chain}

\section{Supply chain}

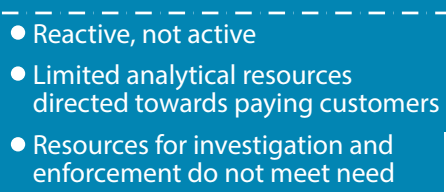

Limited assessment of quality due to cost and lack of laboratory resources

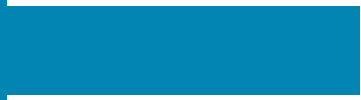

Limited assessment of quality due to limited field testing resources and high cost of lab analysis

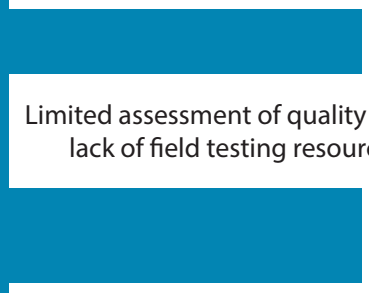

Purchasing decisions are based on price, not quality

\section{Raw Materials}

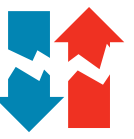

\section{Manufacturer}
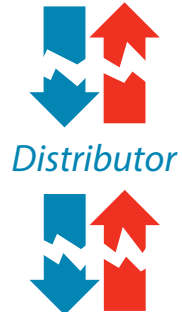

Pharmacy/

Chemist

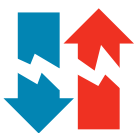

Patients

\section{Effects of broken supply chain}

- Limited post-market

assessment of quality due to

cost and lack of resources

- Poor quality control

- Substitution of raw materials

- Procurement from least expensive suppliers

- Poor access to capital to improve or update manufacturing process

- Non-adherence to good manufacturing practices

- Deliberate falsification

- Cost determines drug purchasing decision

- Lack of regulation of distributers to ensure safe drug storage and handling practices

- Unable to track chain of custody

- Cost determines drug purchasing decisions

- Lack of licensing and safe storage and handling practices

- Medication selection by the patient often based solely on cost

- Limited access to CVD medications

- Adherence not considered a part of supply chain

Patients bear the brunt

of consequences 
Figure 3: Using the Paper Analytical Device (PAD) for rapid field screening of medications

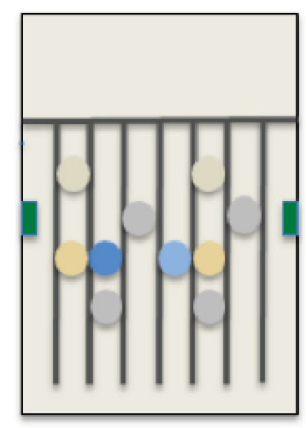

Test card containing preloaded dry reagents

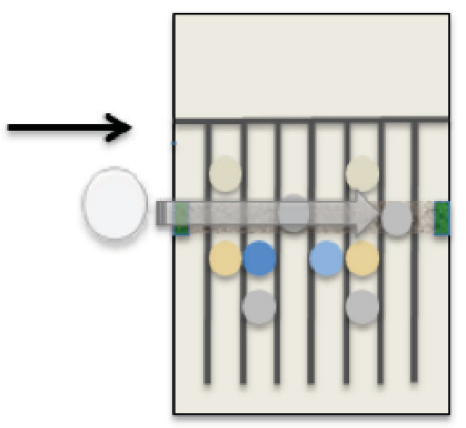

Step 1: Crush tablet and apply to card
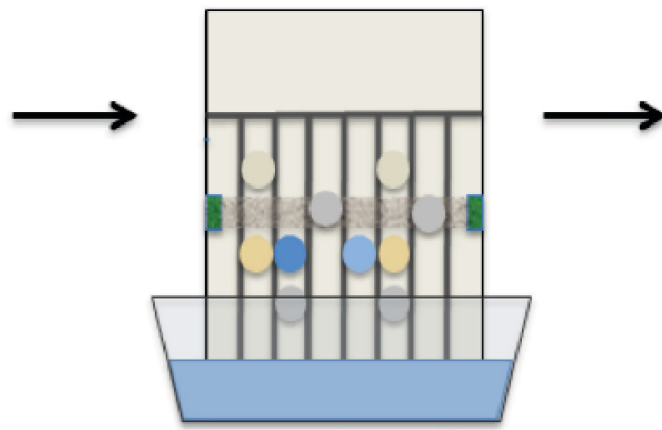

Step 2: Dip card in water for 3 minutes

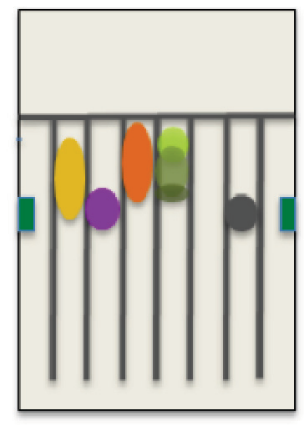

Colors develop in 3 minutes 